\title{
TTR
}

Traduction, terminologie, re?daction

\section{Daniel Vanderveken. Les Actes de discours. Bruxelles, Mardaga, coll. « Philosophie et Langage ", 1988, 226 pages.}

\section{Vera Vidal}

Volume 3, numéro 2, 2e semestre 1990

La traduction des textes sacrés : le domaine biblique

URI : https://id.erudit.org/iderudit/037074ar

DOI : https://doi.org/10.7202/037074ar

Aller au sommaire du numéro

\section{Éditeur(s)}

Association canadienne de traductologie

ISSN

0835-8443 (imprimé)

1708-2188 (numérique)

Découvrir la revue

Citer ce compte rendu

Vidal, V. (1990). Compte rendu de [Daniel Vanderveken. Les Actes de discours. Bruxelles, Mardaga, coll. « Philosophie et Langage », 1988, 226 pages.] TTR, 3(2), 139-145. https://doi.org/10.7202/037074ar d'utilisation que vous pouvez consulter en ligne.

https://apropos.erudit.org/fr/usagers/politique-dutilisation/ 


\title{
La Traduction, la Terminologie et la Rédaction en chronique (4)
}

\author{
Vera Vidal, Antoine Di-Lillo, Jean-Marc Gouanvic
}

NOTA: Depuis le vol. $2, \mathrm{n}^{\circ} 1, T T R$ consacre une chronique régulière aux ouvrages relevant de la traduction, de la terminologie et de la rédaction (et domaines connexes susceptibles d'intéresser les chercheurs de ces disciplines). Les éditeurs qui souhaitent voir leurs publications commentées dans la revue sont priés d'envoyer leurs nouveautés à la rédaction de TTR à l'Université Concordia.

\section{Daniel VANDERVEKEN. Les Actes de discours. Bruxelles, Mardaga, coll. «Philosophie et Langage», 1988, 226 pages.}

On peut affirmer que les fondements de la théorie des actes de discours se trouvent dans la philosophie du langage de Wittgenstein. Déjà dans le Tractatus, ce dernier suggère que la question de la nature de la signification linguistique doit être transformée par l'investigation sur l'usage des expressions. L'analyse de ce que les locuteurs font au moyen du langage devient encore plus poussée dans la période dite "Wittgenstein II», dont les Investigations philosophiques est l'ouvrage le plus représentatif.

Mais ce seront J. Austin et le groupe de l'École d'Oxford (Ryle, Stawson) qui développeront fondamentalement les études des actes de discours en envisageant d'en faire la théorie. Ce courant de la philosophie analytique du langage, connu sous le nom de pragmatisme linguistique, s'est tôt heurté à un grave problème d'ordre méthodologique: comment systématiser de façon rigoureuse ces analyses? La liberté d'action des locuteurs leur permet d'utiliser le même énoncé pour réaliser des actes de parole très diversifiés. Si les notions de signification, de référence et celles qui en dérivent comme l'analyticité, la synonymie, doivent être analysées selon l'usage des expressions dans les différents actes de discours, 
serions-nous en mesure de construire une sémantique générale capable de repérer les traits universaux de l'action linguistique? Ces analyses pourraient-elles mener à la construction d'une sémantique formelle des actes du discours ou même à une pragmatique formelle?

Le philosophe américain John Searle et le logicien et philosophe canadien Daniel Vanderveken se sont unis pour relever ce défi, et réaliser l'ouvrage Foundations of Illocutionary Logic (Cambridge U.P., 1985). D. Vanderveken a réussi à y développer une logique des actes illocutionaires qui fournissaient aux analyses de J. Searle sur les actes de parole («speech acts») la rigueur et la systématisation qui paraissaient, jusque-là, inaccessibles à ces études. D. Vanderveken pousse encore plus loin ces analyses dans Meaning and Speech Acts (en voie de publication chez Cambridge U.P.).

Les Actes de discours est le premier livre en français de l'auteur. C'est un ouvrage au contenu philosophique profond, rigoureux mais informel, qui s'adresse à un vaste public: philosophes, linguistes, logiciens, pédagogues, sémioticiens, ainsi qu'à ceux qui font des recherches en sciences cognitives, en intelligence artificielle, en droit, en théorie de la décision... Il s'agit ici de présenter de façon claire, pédagogique, mais avec rigueur, quelques thèmes, questions et analyses fondamentaux communs à tous ceux qui ont besoin de mieux connaître et de mieux utiliser la théorie des actes de discours.

Après avoir expliqué en quoi consistent les deux grands courants rivaux dans les études de philosophie analytique du langage - le courant logique et celui du langage ordinaire - et le rôle philosophique fondamental qu'il attribue aux actes illocutoires, à savoir celui d'être «l'unité première de signification dans l'usage et la compréhension des langues naturelles» (p. 16), D. Vanderveken énonce le but principal de son ouvrage. Il s'agira de

formuler les principes généraux qui lient les actes de discours à la signification littérale en vue d'intégrer la logique illocutoire et la logique intensionnelle à l'intérieur d'une sémantique formelle générale des langues naturelles capable de caractériser aussi bien les aspects illocutoires que les aspects véri-conditionnels de la signification des énoncés. (p. 8) 
La formulation des principes de cette sémantique aurait pour but d'unifier partiellement la théorie des actes de discours et la sémantique formelle de la vérité. Ainsi, il sera possible «d'interpréter des énoncés de n'importe quel type syntaxique (déclaratif ou non) exprimant des actes illocutoires élémentaires ayant n'importe quelle force illocutoire» (p. 8). Une telle sémantique peut rendre compte de la question des conditions de succès et aussi de vérité des énonciations.

Nombre de philosophes, suivant en cela Frege, s'accordent pour dire que l'unité minimale de signification est l'énoncé et non pas le mot. Mais si l'on s'occupe de l'usage des énoncés, on se rend compte que les unités minimales de signification sont ce qu'Austin appelle «les actes illocutoires». C'est ainsi que, dans le premier chapitre, D. Vanderveken s'occupera de la relation des actes de discours avec la signification. La signification des énoncés contribue à la détermination de la nature des actes illocutoires qui peuvent être accomplis par des énonciations littérales dans tous les différents contextes d'emploi. Il faudra distinguer entre la signification linguistique d'un énoncé en tant que type abstrait et la signification d'une énonciation particulière d'un énoncé dans un certain contexte d'emploi. L'approche de cette sémantique apporte ceci de nouveau qu'elle fournit une «description systématique unifiée de tous les aspects illocutoires et véri-conditionnels de la signification des énoncés» où «la compétence linguistique n'est pas dissociée de la performance» (p. 18). Après la description des types d'énoncés illocutoirement significatifs, l'auteur traite des conditions de succès et de satisfaction des actes de discours, introduisant ainsi le deuxième chapitre où il s'occupera de la présentation de son projet logico-philosophique d'une sémantique générale des conditions de succès et de satisfaction des énonciations.

Le troisième chapitre aborde l'analyse de la forme logique des propositions qui sont à la fois des sens d'énoncés et des contenus d'actes illocutoires. L'auteur y présente un panorama historique des diverses conceptions de la notion de proposition et avance sa définition de propositions comme "porteurs de valeurs de vérité» (puisqu'elles représentent «des états de choses»); il fournit surtout un critère d'identité propositionnelle adéquat pour la théorie des actes de discours, lequel doit être plus fin que l'équivalence logique stricte. Pour que deux propositions de la logique illocutoire soient identiques, elles doivent satisfaire le critère de substitutivité salva felicitate qui dépend des conditions de succès de son énonciation. Ensuite, il énumère les opérations logiques sur les propositions 
qu'on peut effectuer dans le cadre de la sémantique générale: opérations véri-fonctionnelles et modales.

Le quatrième chapitre traite de la notion de force illocutoire, laquelle consiste en un but illocutoire, un mode d'accomplissement de ce but, des conditions sur le contenu propositionnel, des conditions préparatoires, des conditions de sincérité et un degré de puissance; de la définition récursive de l'ensemble de toutes les forces illocutoires; de l'analyse des notions de succès et de satisfaction des énonciations de la sémantique formelle; enfin de la loi d'identité pour les actes illocutoires.

Le cinquième chapitre énumère une série de lois logiques fondamentales qui gouvernent les actes de discours et les énoncés: lois relatives aux buts illocutoires et aux types d'énoncés, aux forces illocutoires et aux marqueurs, lois d'implication.

Le dernier chapitre formule une analyse sémantique des principaux verbes performatifs français. Il s'agit d'une application de l'appareil logique de la sémantique générale. Cette analyse a pour «but de contribuer à la rédaction du dictionnaire raisonné des verbes de parole de la langue française» (p. 167), ce qui va dans le sens de sa conviction - contraire à celle de Saussure qui a établi la distinction langue/parole - selon laquelle l'analyse des actes de discours appartient aux études de la langue. ques fondamentaux:

En conclusion, l'auteur énonce ses présupposés philosophi-

- Quand on utilise le langage de façon sensée, on essaie toujours d'accomplir un acte de discours qui peut soit réussir, soit échouer.

- Les utilisations du langage qui sont réussies obéissent à des règles et une analyse du langage peut aboutir à l'énumération des lois transcendantes fondamentales qui régissent l'usage et la compréhension des langues naturelles et qui concernent des traits linguistiques universels.

- La construction des traits universels du langage qu'il a opérée révèle que la signification des énoncés est attachée à son usage et qu'on ne peut pas séparer la compétence linguistique de sa performance. 
- Il y a une correspondance symétrique entre le langage et le monde.

- Quand on envisage de lier en pensée un contenu propositionnel au monde, on est limité, d'un point de vue transcendental, par les lois qui gouvernent la direction d'ajustement des énonciations. De telles limitations "reflètent des traits essentiels de l'intentionalité linguistique qui sont intériorisés dans l'esprit des locuteurs» (p. 207).

- Les différentes manières dont on peut relier les propositions au monde obéissent, elles aussi, aux limites imposées par les différentes directions possibles d'ajustement des énonciations. Puisqu'il a montré que les déclarations ont la double direction d'ajustement, elles sont les plus fortes parmi les actes illocutoires.

Les déclarations réussies sont ipso facto satisfaites, sincères et sans défaut. C'est pourquoi tout acte illocutoire peut être accompli par le fait de l'accomplissement d'une déclaration alors qu'aucun autre type d'acte illocutoire n'engage à une déclaration. (p. 10)

- La justification de nombreuses lois d'engagement illocutoire est dérivée de l'acceptation de l'un des plus importants principes de la sémantique générale: la rationalité des locuteurs. Il faut supposer qu'ils ont un minimum de cohérence et que, lorsqu'ils réalisent des énonciations réussies, ils s'engagent en même temps avec d'autres actes illocutoires dont les contenus propositionnels sont impliqués fortement par celui qu'ils expriment.

- L'importante contribution de la sémantique générale aux études de philosophie du langage et de l'esprit résulte du fait que les lois fondamentales qui régissent l'usage du langage reflètent les formes a priori de la pensée. La description qu'elle effectue de la structure logique du langage amène à des assertions transcendantales qui fixent des limites à la pensée et à ce qui peut être objet d'expérience humaine dans le monde. Aucun locuteur ne peut transgresser ces règles sans que cela n'entraîne des actes illocutoires non réussis. Les limites que les règles imposent à l'usage du langage fixent indirectement des limites aux contenus de la pensée.

L'énumération de telles conclusions philosophiques régissant les analyses de la sémantique générale ou dérivant de celle-ci prêtent le flanc à de nombreuses discussions. La nature de cette 
présentation de son ouvrage, qui doit être brève et synthétique, nous empêche de discuter en profondeur les innombrables questions qu'il soulève et qui constituent sa valeur fondamentale: impossible de passer à côté des riches problématiques philosophiques et logiques qui sont abordées tout au long de cet ouvrage. Et c'est sans doute le plus grand mérite d'un travail philosophique.

Du fait que nous partions d'autres présupposés de la philosophie du langage, nous ne pouvons prendre à notre compte certaines affirmations, par exemple en ce qui touche la définition des propositions comme véhicules porteurs de valeurs de vérité, ce qui entraine la justification systématique d'autres notions que nous considérons dépourvues de bases théoriques solides, comme celles d'analyticité et de synonymie. De plus, cela suppose une certaine réification de la notion de signification qui aboutira à la formulation de règles qui pourraient donner l'impression qu'on dispose de moyens pour décider de la meilleure traduction parmi plusieurs en conflit. En tant que partisan de la thèse quinéenne de l'indétermination des traductions ${ }^{1}$, nous ne pouvons accepter que l'analyse des actes de parole puisse fournir des critères rigoureux d'identité propositionnelle permettant de décider quel est le meilleur manuel de traduction, quand des manuels adoptent des solutions conflictuelles du point de vue logique mais également justifiées par l'observation du comportement des locuteurs.

Nous avons aussi du mal à accepter sans discussion la théorie de la correspondance telle qu'on la trouve à la base des présuppositions de $\mathrm{D}$. Vanderveken quand il établit les rapports du langage au monde. Même si nous reconnaissons que le langage fixe des limites à tout ce qui peut être pensé et dit sur le monde, nous croyons que les affirmations qui suivent ne sont pas nécessairement déductibles de ses analyses:

[...] le fait que certains actes illocutoires ne puissent en aucun cas être satisfaits impose des limites au monde qui restreignent ce qui peut exister et être objet d'expérience. (p. 10)

Nous admettons que cela limite ce qui peut être objet d'expérience, mais nous croyons qu'affirmer que la satisfaction ou

1. NDLR: Voir l'article de Vera Vidal, «Sur la thèse quinéenne de l'indétermination de la traduction», TTR, III(1), juin 1990. 
la non-satisfaction d'un acte illocutoire puisse limiter ce qui existe est aller trop loin.

[...] le fait que des actes illocutoires de certaines formes aient des conditions de succès ou de satisfaction plus fortes que d'autres montre qu'il existe un ordre a priori de la pensée et du monde. (p. 10)

Nous admettons la relation établie entre les conditions de succès et de satisfaction des actes illocutoires avec les formes $a$ priori de la pensée, mais nous ne sommes pas en mesure d'accepter que de telles conditions révèlent nécessairement un ordre $a$ priori du monde. Il est possible que le monde n'ait aucun principe d'ordre en lui-même et que ce ne soit que nous, en tant qu'être rationnels, qui puissions lui imposer des formes de rationalité.

Ces points de désaccord - parmi d'autres - relatifs aux présupposés théoriques de Daniel Vanderveken et aux nôtres exigeraient de plus amples développements. Il reste que c'est là un travail excellent qui suscite l'admiration pour la rigueur, l'originalité et son apport immense aux recherches dans le domaine de la philosophie, de la logique et de toutes les sciences du langage et de la cognition. Il s'agit d'un ouvrage dont la lecture est obligatoire pour tous ceux qui travaillent dans ces domaines.

Vera Vidal

Université Fédérale de

Rio de Janeiro

Henri G. SCHOGT. Linguistics, Literary Analysis, and Literary Translation. University of Toronto Press, 1988, 177 pages.

L'auteur se penche sur les relations qui existent entre la linguistique, l'analyse littéraire et la traduction littéraire. Plus spécifiquement, il tente de mettre en évidence l'apport de chacune de ces disciplines aux autres. Il indique clairement que le but de l'ouvrage n'est pas de défendre une quelconque théorie, ni de donner une description exhaustive d'un modèle littéraire ou linguistique pour l'analyse de textes. Il vise plutôt à découvrir le rôle joué par 